\title{
The role of engineering in the pursuit of sustainable development: proposal to change the scale of work. The case of the Matanza - Riachuelo Basin
}

\author{
Lafflitto Cristina, Dr. ${ }^{1}$, García Mauricio, Ing. ${ }^{1}$, Armiento Fabián, Ing. ${ }^{1}$, and Porta Andrés, Dr. ${ }^{2}$ \\ ${ }^{1}$ Instituto de Investigaciones en Ingeniería Indutrial I4, Facutaltad de Ingeniería, Universidad Nacional de Lomas de \\ Zamora, Argentina, cristinalafflitto@gmail.com, ing.armiento@gmail.com, mauriciojuliangarcia@gmail.com \\ ${ }^{2}$ Centro Investigaciones Medio Ambientales CIMA, Universidad Nacional de La Plata, aaporta@yahoo.com.ar
}

\begin{abstract}
At present we are faced with the need to minimize the impact that anthropic activities cause on the environment. Therefore, it is essential to achieve sustainable development to achieve a balance between economy, society and nature. That is why the United Nations (UN) establishes this theme as a common objective establishing the Sustainable Development Goals (SDGs) with a target date of 2030.

In this context, innovative solutions are necessary that apply the knowledge of humanity as well as technological capabilities but with a professional ethic that seeks the common benefit. It is at this point that the engineers appear. These professionals acquire planning, resource management and process analysis skills during their training. Thus conforming as important actors of interdisciplinary groups that contribute to improve the environmental quality.

In this work, the results achieved in the Lafflitto engineering doctoral thesis (2017) in the search for sustainable development in the Matanza Riachuelo Basin (CMR), an emblematic case of environmental degradation in the Argentine Republic, were related. Along with the generic and specific competences for the case of industrial engineering proposed by the Federal Council of Deans of Engineering (CONFEDI). Analyzing the possibility of applying the skills acquired on a regional scale.
\end{abstract}

Keywords: Engineering, Sustainable Development, Competencies, Matanza-Riachuelo Basin, regional scale.

Digital Object Identifier (DOI):

http://dx.doi.org/10.18687/LACCEI2020.1.1.636

ISBN: 978-958-52071-4-1 ISSN: 2414-6390

18 $^{\text {th }}$ LACCEI International Multi-Conference for Engineering, Education, and Technology: "Engineering, Integration, and Alliances for a Sustainable Development" "Hemispheric Cooperation for Competitiveness and Prosperity on a Knowledge-Based Economy", July 27-31, 2020, Virtual Edition. 


\title{
El rol de la ingeniería en la consecución del desarrollo sostenible: propuesta de ampliación de campo de acción. Estudio de caso: la sostenibilidad en la Cuenca Matanza - Riachuelo.
}

\author{
Lafflitto Cristina, Dr. ${ }^{1}$, García Mauricio, Ing. ${ }^{1}$ Armiento Fabián, Ing. ${ }^{1}$, and Porta Andrés, Dr. ${ }^{2}$ \\ ${ }^{1}$ Instituto de Investigaciones en Ingeniería Indutrial I4, Facutaltad de Ingeniería, Universidad Nacional de Lomas de Zamora, \\ Argentina, cristinalafflitto@gmail.com, ing.armiento@gmail.com, mauriciojuliangarcia@gmail.com \\ ${ }^{2}$ Centro Investigaciones Medio Ambientales CIMA, Universidad Nacional de La Plata, aaporta@yahoo.com.ar
}

\begin{abstract}
Resumen-En la actualidad nos encontramos ante la necesidad de minimizar el impacto que las actividades antrópicas causan en el ambiente. Por lo cual es imprescindible conseguir el desarrollo sostenible para lograr el equilibrio entre economía, sociedad y naturaleza. Es por ello que la Organización de Naciones Unidas (ONU) establece esta temática como un objetivo común estableciendo los Objetivos de Desarrollo Sostenible (ODS) con plazo de consecución el año 2030.

En este contexto, son necesarias soluciones innovadoras que apliquen el conocimiento de la humanidad así como las capacidades tecnológicas pero con una ética profesional que busque el beneficio común. Es en este punto donde aparecen los ingenieros. Estos profesionales adquieren durante su formación capacidades de planificación, gestión de recursos y análisis de procesos. Conformándose así como actores importantes de grupos interdisciplinarios que contribuyan a mejorar la calidad ambiental.

En el presente trabajo se relacionaron los resultados alcanzados en la tesis de doctorado de la ingeniería Lafflitto (2017) en la búsqueda del desarrollo sostenible en la Cuenca Matanza Riachuelo (CMR), un caso emblemático de degradación ambiental de la República Argentina. Junto con las competencias genéricas y específicas para el caso de la ingeniería industrial propuestas por el Consejo Federal de Decanos de Ingeniería (CONFEDI). Analizando la posibilidad de aplicar las competencias adquiridas en una escala regional, lo que implicaría una ampliación en el campo de acción tradicional del ingeniero.
\end{abstract}

Palabras Claves-Ingeniería, Desarrollo Sostenible, Competencias, Cuenca Matanza- Riachuelo, escala regional.

\section{INTRODUCCIÓN}

A. Desarrollo sostenible y ODS.

Ante la alta degradación del ambiente a nivel global surgió el concepto de desarrollo sostenible exponiendo que no es posible pensar solo en el desarrollo económico ya que necesitamos para esto recursos naturales y a la sociedad.

El desafío que genera el logro de ese necesario equilibrio de vino en el establecimiento de los ODS, 17 objetivos que tienen un fin común: conseguir el desarrollo equilibrado de la sociedad, el ambiente y la economía [1]. Esta herramienta desarrolla a través de 169 metas una interrelación entre diversas problemáticas ambientales que cada objetivo ataca en particular. Esto provoca un efecto en cadena es decir que una meta conseguida contribuye a la realización de otra meta. Una

Digital Object Identifier (DOI):

http://dx.doi.org/10.18687/LACCEI2020.1.1.63]

ISBN: 978-958-52071-4-1 ISSN: 2414-6390 vez este set de objetivos demuestra y pone en valor la importancia de integrar los 3 pilares ya mencionados: económico, social y ambiental [2].

\section{B. La problemática ambiental en la CMR}

La CMR, la más contaminada de la Argentina [3], La cuenca hidrográfica Matanza-Riachuelo (CMR) se extiende cubriendo una superficie de $2.238 \mathrm{~km} 2(0,1 \%$ del territorio nacional) incluyendo parte de la Ciudad Autónoma de Buenos Aires (CABA) además de otras 14 jurisdicciones de la Provincia de Buenos Aires. Desde su nacimiento el río se denomina Matanza, y a partir del Puente de la Noria (sobre la Av. Gral. Paz, que delimita la CABA) y hasta su desembocadura en el Río de la Plata adopta el nombre de Riachuelo. Sin embargo, la población radicada actualmente en ella -de más de 5 millones de habitantes- representa el $13,5 \%$ de la población total del país (Figura 1) [4].

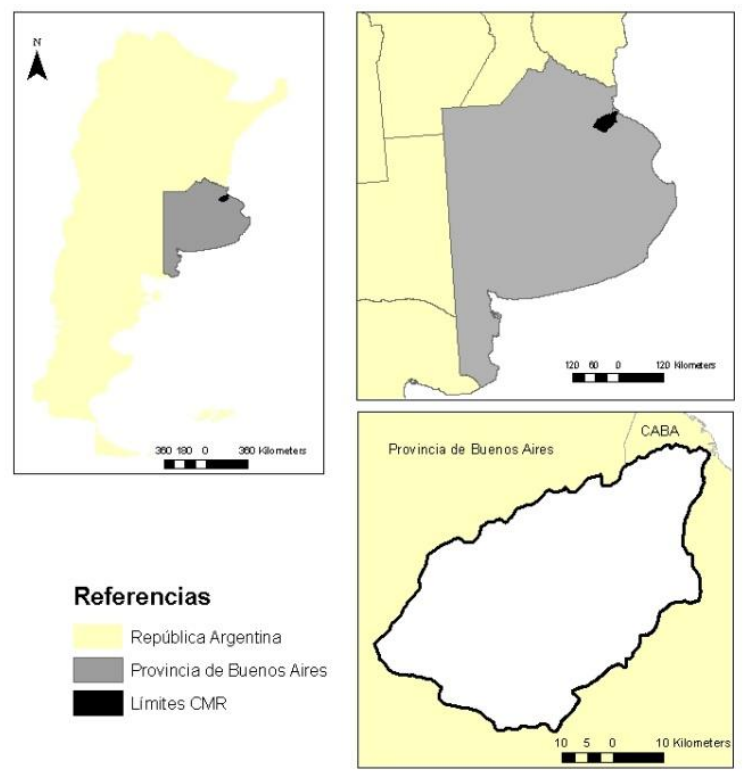

Fig. 1 Ubicación de la CMR. Fuente: Lafflitto 2017 [4].

Este es un caso emblemático de degradación ambiental para la Argentina, dada la historia de intentos fallidos de saneamiento, incrementos de impactos ambientales tales como: aumento de

18th LACCEI International Multi-Conference for Engineering, Education, and Technology: "Engineering, Integration, and Alliances for a Sustainable Development" "Hemispheric Cooperation for Competitiveness and Prosperity on a Knowledge-Based Economy", July 27-31, 2020, Virtual Edition. 
la urbanización sin planificación, pérdida de espacios de alta calidad agropecuaria, ineficiente sistema de saneamiento cloacales y provisión de agua potable, entre otros [4].

\section{Las competencias de la ingeniería y los estudios ambientales.}

En la actualidad, Argentina está transitando por un camino de modificación de los planes de estudio de las carreras de ingeniería. El objetivo de estas modificaciones es formar mejores profesionales para las necesidades futuras. Es por ello que la formación por competencias aparece con fuerza.

Desde el CONFEDI se presentan las competencias generales tanto técnicas como sociales, políticas y actitudinales que cualquier ingeniero debiera adquirir a lo largo de su formación [5]. Además, se genera un propuesta de competencias específicas para cada rama de la ingeniería en el denominado Libro Rojo [6].

TABLA 1

EJEMPLOS DE CONOCIMIENTOS ADQUIRIDAS POR UN INGENIERO INDUSTRIAL Y SUS IMPLICANCIAS EN TEMÁTICAS AMBIENTALES REGIONALES. ADAPTADO: LAFFLITTO \& ZULETA 2012.

\begin{tabular}{|c|c|c|}
\hline Conocimiento & Aplicación Actual & Aplicación Regional \\
\hline Estadística & Estadística de fallas & $\begin{array}{lr}\begin{array}{l}\text { Análisis } \\
\text { para }\end{array} & \text { zonifivariados } \\
\text { territorial } & \\
\end{array}$ \\
\hline Procesos lógicos & Proceso de producción & $\begin{array}{l}\text { Proceso de intercambio } \\
\text { de energía en cuencas } \\
\text { hídricas }\end{array}$ \\
\hline $\begin{array}{l}\text { Herramientas } \\
\text { Planificación }\end{array}$ & $\begin{array}{l}\text { Desarrollo de plan de } \\
\text { producción }\end{array}$ & $\begin{array}{l}\text { Desarrollo del plan de } \\
\text { ordenamiento ambiental }\end{array}$ \\
\hline $\begin{array}{l}\text { Herramientas } \\
\text { Diseño }\end{array}$ & $\begin{array}{l}\text { Diseño de plantas } \\
\text { industriales en Auto } \\
\text { CAD }\end{array}$ & $\begin{array}{l}\text { Generación de mapas } \\
\text { temáticos en SIG/GIS }\end{array}$ \\
\hline Lay outs & $\begin{array}{l}\text { Ordenamiento de } \\
\text { equipamientos en una } \\
\text { planta }\end{array}$ & $\begin{array}{l}\text { Ordenamiento de usos en } \\
\text { el territorio }\end{array}$ \\
\hline $\begin{array}{l}\text { Generación } \\
\text { Indicadores }\end{array}$ & Indicadores de calidad & $\begin{array}{lll}\text { Indices de calidad } \\
\text { ambiental }\end{array}$ \\
\hline Logística & $\begin{array}{l}\text { Red de transporte de } \\
\text { materiales }\end{array}$ & $\begin{array}{l}\text { Modelos de transporte } \\
\text { inter e intra ciudades }\end{array}$ \\
\hline Ingeniería Ambiental & $\begin{array}{l}\text { Estudio de impacto } \\
\text { ambiental }\end{array}$ & $\begin{array}{l}\text { Estudio de impacto } \\
\text { ambiental }\end{array}$ \\
\hline Innovación & $\begin{array}{l}\text { Investigación y } \\
\text { desarrollo para generar } \\
\text { nuevos productos }\end{array}$ & 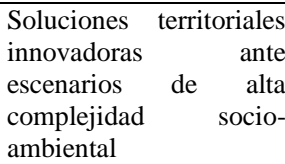 \\
\hline Investigación operativa & $\begin{array}{l}\text { Necesidad de personal } \\
\text { de atención al cliente }\end{array}$ & $\begin{array}{l}\text { Necesidades de espacio } \\
\text { para usos particulares en } \\
\text { la ciudad como por } \\
\text { ejemplo para } \\
\text { aparcamiento vehicular }\end{array}$ \\
\hline
\end{tabular}

Es fundamental destacar que en las competencias se formaliza el campo de acción de un ingeniero industrial tanto privado como público. En gran parte el tipo de proyectos territoriales/ambientales son de carácter público, o una combinación público-privado.
Es por esto que la importancia de la ejecución de proyectos como el que plantea el caso de estudio son relevantes.

Se observó, al analizar los conocimientos que adquiere un ingeniero industrial durante su formación académica, que muchos de ellos son aplicables tanto en la escala industrial como en la escala regional. En la Tabla 1 se ejemplifican algunos conocimientos con sus aplicaciones en cada escala menciona.

De este análisis surge la pregunta que guía este trabajo: ¿es factible que un ingeniero, en particular un ingeniero industrial, pueda ampliar su campo de acción a la escala regional y desarrollar propuestas que impliquen una mejora en la calidad ambiental de un Sistema natural? El presente trabajo tiene por objetivo realizar un análisis cualitativo de un caso de estudio que permita determinar la interrelación entre las competencias planteadas para los ingenieros industriales y las competencias necesarias para realizar aportes en la búsqueda de soluciones tendientes al desarrollo sostenible.

\section{METODOLOGÍA}

El presente trabajo se basó en el estudio de caso, realizando un análisis cualitativo donde se estudiaron las competencias genéricas de un ingeniero propuestas por CONFEDI [5] realizando una lista de chequeo verificando cuales de estas se reflejaban en los resultados obtenidos y los procesos de obtención de estos en la tesis doctoral realizada por la ingeniera Cristina Lafflitto. Este desarrollo, titulado "Gestión sustentable del Periurbano: una metodología estratégica como aporte para el incremento de la calidad ambiental en la Cuenca MatanzaRiachuelo desde la planificación territorial" [4], toma como objeto de estudio un Sistema natural con límites físicos el cual, cómo se describió en la introducción, es un caso de extrema degradación ambiental. Además, al tratarse de un desarrollo realizado por una ingeniería industrial, se realice el mismo procedimiento tomando de base para el análisis las competencias específicas propuestas por CONFEDI en su Libro Rojo [6].

\section{RESULTADOS}

Luego del análisis, se detectaron las siguientes competencias que han sido demostradas en la tesis de la ingeniería Lafflitto [4]. Se detalla en los siguientes apartados.

\section{A. Competencias genéricas tecnológicas \\ 1) Identificar, formular y resolver problemas de ingeniería}

El desarrollo de la tesis en si misma comprende esta competencia. A lo largo de la misma se identifican, formulan e se acercan posibles soluciones a diversas problemáticas ambientales de la CMR. Las mismas se podrían englobar en la problemática ambiental de los usos de suelo no planificados dentro del Sistema. Se trabaja en todo el desarrollo en una variable novedosa, sobre todo para la ingeniería industrial que es la territorial. 
En la Figura 2, a modo de ejemplo, se observa el modelo generado para explicar el incremento de la población en riesgo ambiental, uno de los problemas que presenta la CMR.

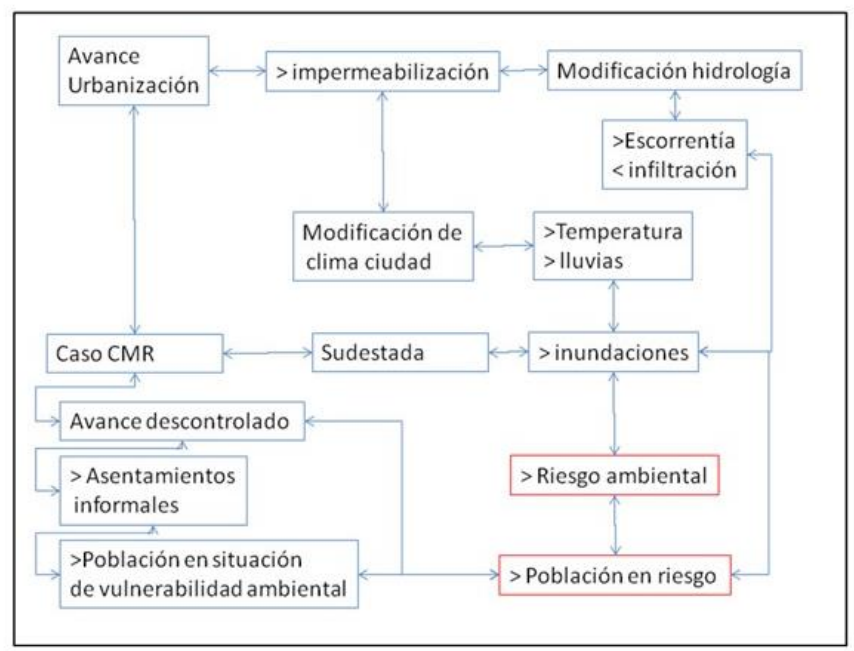

Fig. 2 Modelo de causas población de riesgo CMR. Fuente: Lafflitto 2017 [4].

2) Concebir, diseñar y desarrollar proyectos de ingeniería y 3) Gestionar -planificar, ejecutar y controlar- proyectos de ingeniería

La realización de este tipo de investigaciones puede considerarse en sí misma un Proyecto. Desarrollar un marco teórico, plantear objetivos, metodologías a utilizar, generar resultados y analizarlos para poder sacar conclusiones estarían corroborando las competencias genéricas del ítem 2).

4) Utilizar de manera efectiva las técnicas y herramientas de la ingeniería.

La capacidad de identificar, seleccionar y utilizar herramientas se ve claramente en el proceso que fue llevado acabo para realizar los mapas de usos de suelo (Figura 3). Para poder generar el mismo se identificaron numerosas herramientas y metodologías seleccionando/desarrollando una Ad Hoc. Esto debido a la previa identificación del uso periurbano como un uso estratégico a gestionar para lograr un acercamiento al desarrollo sostenible. Fue por esto que el mapa de uso de suelo se generó mediante interpretación visual de imagines satelitales, junto a interpretación de firmas espectrales y validación a campo. De esta manera se pudo localizar el periurbano sin perder sus características.

5) Contribuir a la generación de desarrollos tecnológicos y/o innovaciones tecnológicas

Es en esta competencia en particular donde se observó el fuerte de la tesis. En la misma se observa por un lado la detección de necesidades insatisfechas y nuevas maneras de satisfacerlas mediante soluciones tecnológicas, en lo que respecta a la detección del uso periurbano mediante la metodología comentada en el apartado 4) más otra metodología utilizada de manera innovadora para detectar el periurbano a escala regional. Ya que diversos autores especialistas en periurbano describían la dificultad de ubicarlo espacialmente.

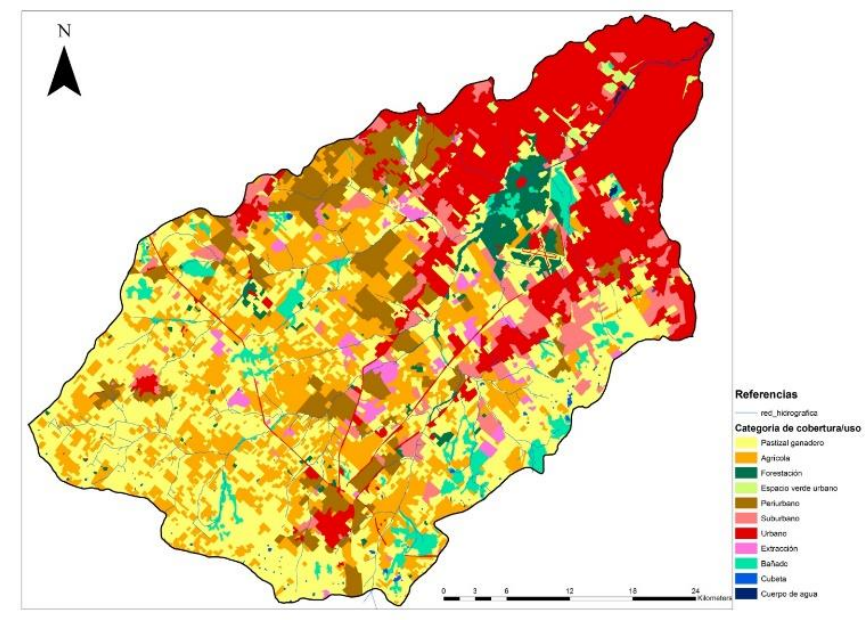

Fig. 3 Mapa usos de suelo 2009. Fuente: Lafflitto 2017 [4].

Logrando además una clasificación de tipos de periurbano en la escala local que permite observar la evolución temporal de los usos de suelo hacia el uso urbano (Figura 4).

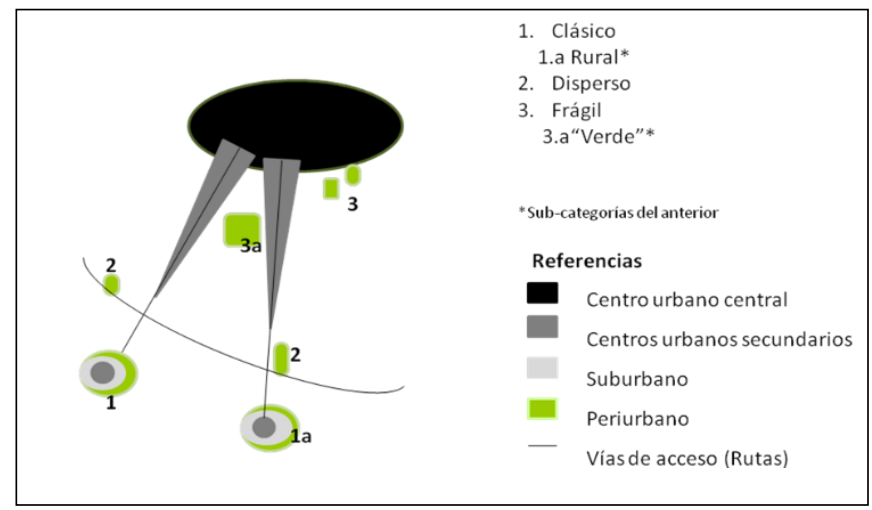

Fig. 4 Modelo conceptual de la tipología de uso periurbano para la CMR a escala local. Fuente: Lafflitto 2017 [4].

Además, se observó una importante utilización creativa de tecnologías/conocimiento disponible al proponer utilizar la norma internacional ISO 14001 para la gestión ambiental cuyo ámbito de aplicación hasta el momento era el de industrias, empresas e instituciones públicas o privadas. En el trabajo se propone utilizar esta herramienta ampliamente validada en una escala novedosa: la territorial (Figura 5).

\section{B. Competencias genéricas sociales, políticas y actitudinales.}


Dentro de este grupo de competencias existen algunas que se evidencian en resultados concretos:

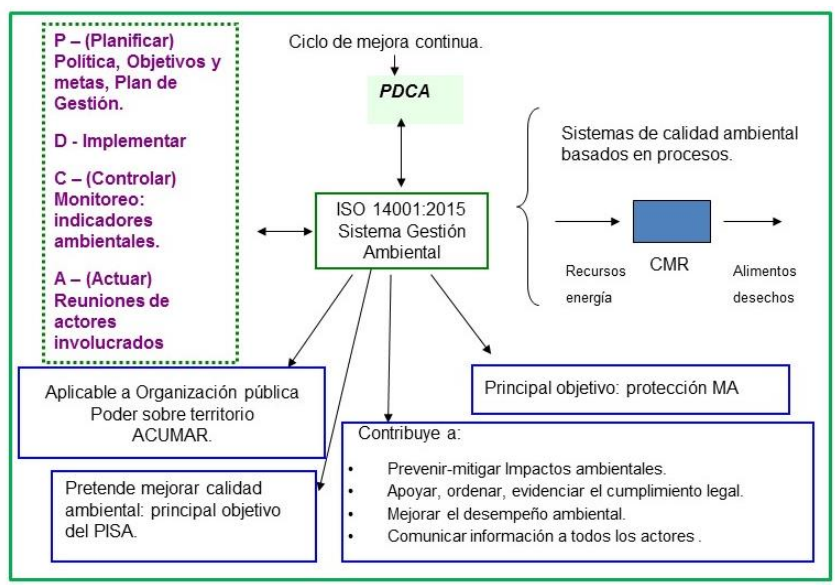

Fig. 5 Por qué aplicar ISO 14001 en la CMR. Adaptado de Lafflitto 2017 [4].

\section{1) Desempeñarse de manera efectiva en equipos de trabajo}

El desarrollo de proyectos ambientales territoriales que busquen la propuesta de soluciones factibles difícilmente puedan realizarse en soledad. Es necesaria la interacción con diversos profesionales de diferentes temáticas que aporten al análisis del Proyecto conceptos, metodologías y teorías que por sí solo un ingeniero no podría tener. En el caso de estudio esto se ve reflejado en varios puntos desarrollados, pero sobre todo en la generación de un capítulo del libro "Rehabilitación de ambientes perdidos" [7] donde participaron: geólogos, arqueólogos, biólogos, sociólogos, e ingenieros.

2)Actuar con ética, responsabilidad profesional y compromiso social, considerando el impacto económico, social y ambiental de su actividad en el contexto local y global.

En sí mismo el tema del caso de estudio muestra un compromiso social que considera el impacto ambiental de las actividades antrópicas en la CMR buscando proponer estrategias que logren tender al desarrollo sostenible del Sistema. Tal como se puede observar en la Figura 6, que resume la propuesta a la que se llega como conclusión de todos los resultados generados.

Otras pueden verse resueltas en el mismo desarrollo de una tesis doctoral, su documento y su defensa:

\section{1) Comunicarse con Efectividad;}

\section{2) Aprender en forma continua y autónoma;}

3) Actuar con espíritu Emprendedor.

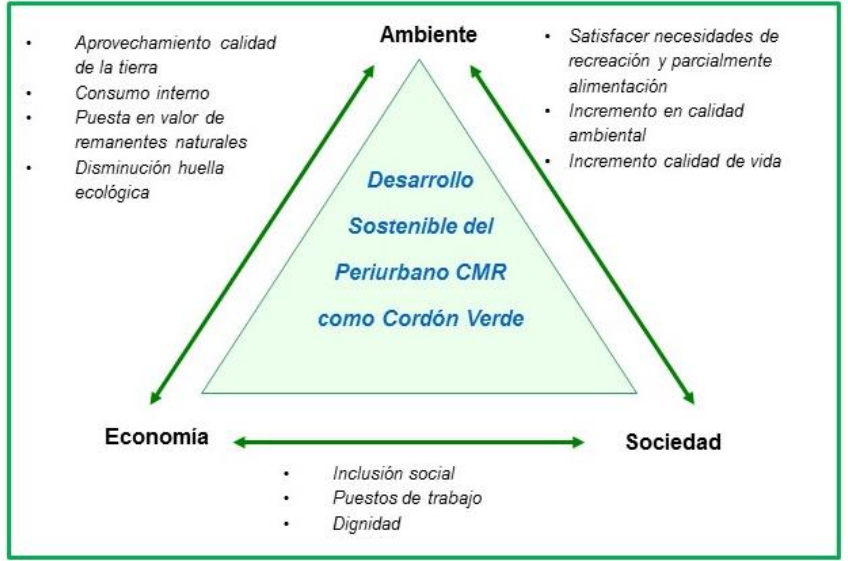

Fig. 6 Sinergia entre los ejes del Desarrollo sostenible mediante la propuesta. Fuente: Lafflitto 2017 [4].

\section{Competencias específicas ingeniería industrial.}

En la formación del ingeniero industrial tiene la particularidad de que sus competencias específicas están relacionadas con evaluar la sostenibilidad ambiental de un proyecto, minimizar los impactos ambientales, proyectar y gestionar todo lo relacionado a estos. Es aquí donde encontramos una interrelación entre las competencias y la posibilidad de aportar en la búsqueda de soluciones ambientales en proyectos que exceden al ámbito indsutrial.

\section{CONCLUSIÓN Y DISCUSIÓN}

La modificación de los planes de estudios según las recomendaciones del CONFEDI se están realizando en un momento clave para el ambiente. Justamente por ello la Agenda 2030 de ONU es una herramienta que se está explorando en el grupo de investigación de la Facultad de Ingeniería de la UNLZ para involucrar cada vez más profesionales de la ingeniería en temáticas ambientales, así como relacionarse de una forma sinérgica con otros grupos de profesionales de manera tal de analizar la problemática ambiental de una forma integral y transversal a todas las actividades (Figura 7).

Este nuevo punto de vista presenta grandes desafíos para el ámbito académico, en especial para las Universidades y su cuerpo docente. Es imprescindible el cambio de paradigma, debe dejarse atrás la visión del ingeniero industrial como un profesional basado en la técnica para verlo como un profesional integral capaz de generar conocimiento.

Si bien el método científico es abordado en la currícula, debe considerarse este como una herramienta que debe ser aplicada durante el proceso de formación. En este sentido, es relevante la inserción de los estudiantes en proyectos de investigación para que aprendan a desenvolverse en este ámbito eficazmente. Sería conveniente generar una red de centros de investigación entre universidades para que los estudiantes puedan participar 
de grupos interdisciplinarios y adquirir así una visión integral. Debería ser obligatoria la realización de una tesis de grado, tanto en aspectos clásicos de ingeniería como orientados a la investigación aplicada, ya sea tecnológica y/o científica. Todos estos puntos estarán reforzados ante la nueva mirada por competencias.

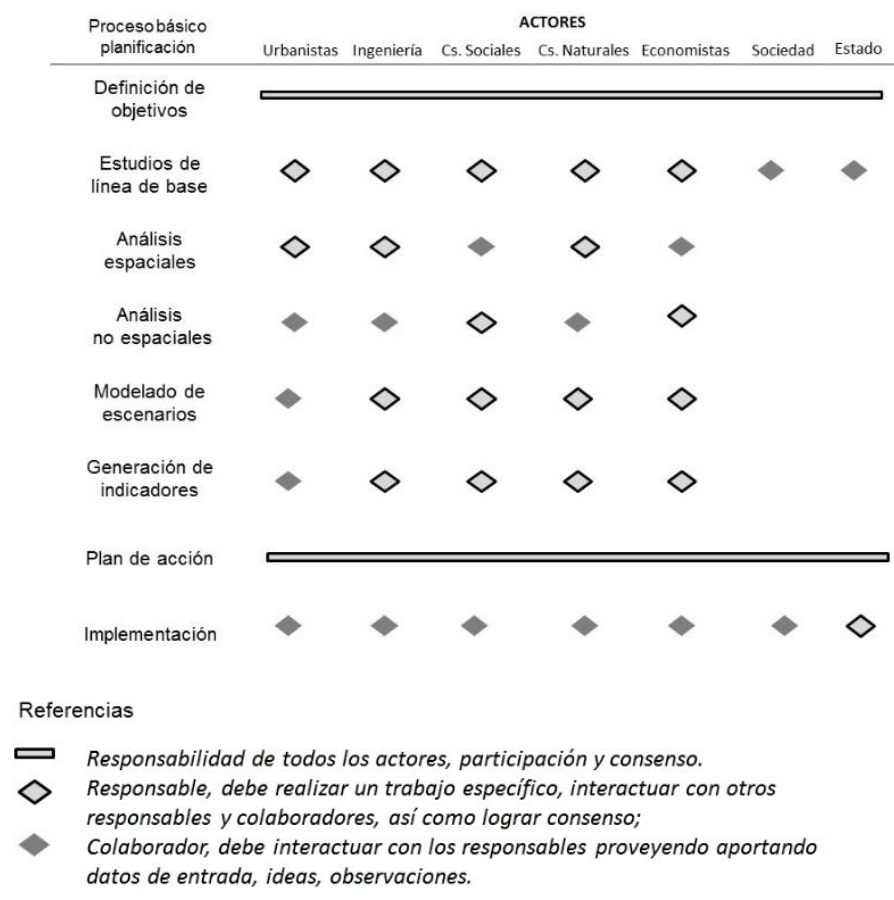

Fig. 7 Interacciones entre diferentes actores en un proceso de planificación considerando trabajo interdisciplinario. Adaptado: Lafflitto \& Zuleta [8].

Estas modificaciones ampliarán la visión analítica del graduado, posibilitando un mejor desempeño en trabajos tradicionales, así como una apertura hacia la aplicación de sus conocimientos en pos de contribuir a la resolución de conflictos ciudad-naturaleza y el acercamiento al desarrollo sostenible. Es aquí donde debe focalizarse en el bien común que será la consecución generación/creación de una ciudad compatible con el ambiente con un entorno que posea usos compatibles con las capacidades naturales del área. Por otra parte, debe debatirse cual cuál es el punto de convergencia entre las necesidades reales de consumo y la bio-capacidad del ambiente en el que vivimos [8].

Es interesante el resultado de un análisis FODA realizado (Figura 8), justamente se ve lo detallado anteriormente en cuanto a los factores que se verán fortalecidos ante la implementación de los nuevos planes de estudio.

Este trabajo se trata sobre un caso de estudio, por lo cual el análisis que se realizó es del tipo cualitativo. Sería interesante continuar esta línea de investigación estudiando otros casos para poder realizar un análisis cualitativo, lo que permitirá definir estrategias para su efectiva potencialización.
TABLA 2

ANÁLISIS FODA DEL ROL DE LOS INGENIEROS EN PROYECTOS PARA EL DESARROLLO SOSTENIBLE A ESCALA REGIONAL. ADAPTADO: LAFFLITTO \& ZULETA [8].

\begin{tabular}{|l|l|}
\hline \multicolumn{1}{|c|}{ Fortalezas } & \multicolumn{1}{c|}{ Debilidades } \\
\hline $\begin{array}{l}\text { Alto dominio de herramientas de } \\
\text { planificación }\end{array}$ & $\begin{array}{l}\text { Baja interacción con otras ramas del } \\
\text { conocimiento, trabajo } \\
\text { interdisciplinario }\end{array}$ \\
\hline $\begin{array}{l}\text { Alto manejo de sistemas y } \\
\text { variables }\end{array}$ & Escasa intervención en proyectos \\
\hline \multicolumn{1}{|c|}{ Oportunidades } & \multicolumn{1}{c|}{ Amenazas } \\
\hline $\begin{array}{l}\text { Alta necesidad de planificación } \\
\text { eficiente en la temática socio- } \\
\text { ambiental }\end{array}$ & $\begin{array}{l}\text { Mayores beneficios en el ámbito } \\
\text { privado }\end{array}$ \\
\hline $\begin{array}{l}\text { Alta necesidad de soluciones } \\
\text { innovadoras para el desarrollo } \\
\text { sustentable }\end{array}$ & $\begin{array}{l}\text { Visión de contraposición de } \\
\text { intereses entre los problemas } \\
\text { ambientales y el ámbito industrial }\end{array}$ \\
\hline
\end{tabular}

\section{AGRADECIMIENTOS}

Agradecemos los recursos y gestiones institucionales provistas por el Instituto de Investigaciones en Ingeniería Industrial (I4), instituto asociado de la Comisión de Investigaciones Científicas de la Provincia de Buenos Aires (CIC).

Agradecemos a la Agencia Nacional de Promoción de Ciencia y Técnica (ANPCyT) quien mediante su programa de temas estratégicos financió gran parte de los trabajos realizados en la tesis mediante subsidios PFDT, PICT y PME.

\section{REFERENCIAS}

[1] Naciones Unidas, La Agenda 2030 y los Objetivos de Desarrollo Sostenible: una oportunidad para América Latina y el Caribe (LC/G.2681-P/Rev.3), Santiago, 2018.

[2] Figueira A., M. García y C. Lafflitto, Objetivos de desarrollo sostenible (ODS): una estrategia para mejorar la gestión ambiental de las industrias de la región, XII Congreso Argentino de Ingeniería Industrial, UTN, Santa Cruz, 2019.

[3] Faggi, A. \& Lafflitto, C. "La Cuenca Matanza-Riachuelo. Una mirada ambiental para recuperar sus riveras". Manual. Español. Argentina. 2014.

[4] Lafflitto, C. "Gestión sustentable del Periurbano: una metodología estratégica como aporte para el incremento de la calidad ambiental en la Cuenca Matanza-Riachuelo desde la planificación territorial.", Lomas de Zamora, Facultad de Ingeniería, U.N.L.Z., 2017.

[5] CONFEDI. "Libro Rojo", Mar del Plata, Universidad Fasta Ediciones, 2014.

[6] CONFEDI. "Propuestas de estandares de $2^{\circ}$ generación para la acreditación de las carreras de ingeniería en la Republica Argentina", Mar del Plata, Universidad Fasta Ediciones, 2018.

[7] Zuleta, G.A., Guida Johnson, B., Lafflitto, C.M., Faggi, A.M., De Magistris, A.A., Tchilinguirian, P., Weissel, M., y Zarrilli, A.G. (2012) Rehabilitación de ambientes perdidos en mega-ciudades: el caso de la cuenca Matanza-Riachuelo. Capítulo XX. En: Paisajes perdidos (J. Athor, Ed.). Fundación Azara. P. 445-459.

[8] Lafflitto, C. \& Zuleta, G. "El rol de la ingeniería industrial en la planificación ambiental de ciudades. De la escala industrial a la regional", V Congreso Argentino de Ingeniería Industrial, Lomas de Zamora, Noviembre 2012. 